\title{
Análisis ambiental: impactos generados por los residuos agrícolas en el municipio de El Dorado (Meta, Colombia)
}

\section{Environmental analysis: impacts generated by agricultural waste in the municipality of El Dorado (Meta, Colombia)}

\author{
CASTRO-GARZÓN, Hernando ${ }^{1}$ \\ CONTRERAS, Erika J. ${ }^{2}$ \\ RODRÍGUEZ, Juan P. ${ }^{3}$
}

\begin{abstract}
Resumen
Este trabajo analizó el impacto ocasionado por las labores agrícolas del municipio del Dorado en el departamento del Meta, debido a la disposición de residuos inertes y biomasa. Se identificarón los cultivos a través de un estudio explicativo estableciendo los factores ambientales afectados mediante la matriz de Leopold; adicionalmente a través de un instrumento cuantitativo se recolectó información para identificar los mayores generadores de los residuos mencionados y las prácticas que producían efectos nocivos para el entorno.

Palabras clave: sostenibilidad, impacto ambiental, residuos de cosechas.
\end{abstract}

\begin{abstract}
This work analyzed the impact caused by the agricultural work of the municipality of Del Dorado in the department of Meta, due to the provision of inert waste and biomass. Crops were identified through an explanatory study establishing the affected environmental factors through Leopold's matrix; in addition, information was collected through a quantitative instrument to identify the largest generators of the above-mentioned waste and practices that produced harmful effects on the environment.

Key words: sustainability, environmental impact, crop residues.
\end{abstract}

\section{Introducción}

La sociedad se preocupa por la generación de desechos debido a la clara disminución de los recursos disponibles para el consumo humano. Como consecuencia del crecimiento de la población mundial como lo expresa la Organización de las Naciones Unidas (ONU, 2011) "la población mundial para el año de 1950 era aproximadamente de 2600 millones de personas, y para el 2015, 65 años después, este número se superó casi 3 veces llegando a 7300 millones de habitantes"., convirtiéndose en una restricción para el acceso a los recursos.

\footnotetext{
${ }^{1}$ Profesor, Escuela de Administración y Negocios. Facultad de Ciencias Económicas, Universidad de los Llanos, Villavicencio, Colombia. Correo electrónico: hcastro@unillanos.edu.co

${ }^{2}$ Administradora de Empresas. Universidad de los Llanos, Villavicencio, Colombia.

Correo electrónico: erika_julieth26hotmail.com

${ }_{3}$ Profesor Titular. Facultad del Medio Ambiente y Recursos Naturales. Universidad Distrital Francisco José de Caldas. Bogotá, Colombia. Correo electrónico: jprodriguezm@udistrital.edu.co
} 
Como resultado del desarrollo, "el perfeccionamiento histórico del hombre trajo consigo el aumento en la generación de residuos de toda clase y con ello diferentes tipos de enfermedades y males debido a su inadecuada forma de disposición y vertimiento" (Grande, 2016, p.13) estableciendo que a medida que la población consume carece de consciencia respecto a los impactos negativos provocados por la producción de residuos y la ausencia de capacidad de su manejo. Se definen como, "un producto cuya característica fundamental es que no tiene valor ni estimación en las circunstancias en las que se genera y que se ha de retirar para facilitar o mejorar los procesos" (Cuadros, 2008, p.5).

Los residuos se clasifican de acuerdo con su origen en los sectores de producción, como lo afirma (Bernal \& Gordan, 2008, p. 12), "primarios o de producción de materias primas, secundarios o industriales y terciarios o de servicios". En este caso, se trabajó con el sector primario: residuos agrícolas, definido por (Lopez \& Boluda, 2014, p. 493) como "una fuente de materia orgánica exógena que pueden volver al suelo con objeto de ser utilizados, mejorar el desarrollo de los cultivos y aumentar la calidad del suelo.", donde estos, poseen una función específica en el ciclo productivo de las cosechas si son utilizados de forma adecuada.

Ahora bien, "a nivel mundial se generan grandes toneladas de residuos agrícolas que son desechados y actúan como foco de contaminación ambiental; en muy pocos lugares se les da un uso productivo y se le atribuye un valor añadido" (Figueredo et al., 2011, p. 16); pudiendo constituirse como fuente de energía en el sistema productivo que conforma, en su lugar se genera grandes volúmenes de entropía.

Es una práctica común en las explotaciones según (Porras \& González, 2016, p.93) que "en las actividades agrícolas no se realiza la disposición de residuos en un relleno o zona adecuada, siendo la opción más económica la quema descontrolada del material" produciendo emisión de gases de efecto invernadero. Esto a pesar de ser una oportunidad para encontrar nuevas alternativas que disminuya los costos para los agricultores y el impacto ambiental por su mal manejo, tal como lo expresa (Piña, Nieto, \& Robles, 2016, p. 145) "en Iberoamérica, en los últimos años, se han visto incrementados los estudios para la utilización de distintos residuos agrícolas".

A continuación, se mencionan los 4 tipos de residuos agrícolas existentes (Coronado \& Valencia, 2015, p. 14) "fitosanitarios, fertilizantes, biomasa residual e inertes", aunque en la producción de residuos agrícolas son en su mayoría dos tipos: los de biomasa residual, los cuales son "residuos de composición química parecida, son renovables puesto que provienen de seres vivos, se generan en grandes cantidades y están muy dispersos en el espacio" y los residuos inertes que son "los materiales empleados en la actividad agrícola que queda como residuo y tienen potencial para contaminar el suelo y las aguas" (Coronado \& Valencia, 2015, p.14).

Para Colombia, "la producción agrícola es uno de los pilares económicos para el desarrollo del país" según (Coronado \& Valencia, 2015, p. 15) representando un importante porcentaje territorial. Así lo refleja la Encuesta Nacional Agropecuaria al decir que "el área total cultivada fue 5.121.508 ha, el grupo de cultivos que mayor participación tuvo fueron los cultivos agroindustriales con una participación de 32,4\%, registrando un área de 1.658.598 ha" (DANE, 2016).

En el departamento del Meta, "se tiene una tradición agrícola sobresaliente en diferentes productos como: la palma africana, arroz, frutales y plátano, generando grandes cantidades de residuos heterogéneos que representan un problema desde el punto de vista ambiental debido a la falta de técnicas para su aprovechamiento y re-uso" (Nuñez, 2012, p. 143). Al adolecer de prácticas de manejo y tratamiento para dichos residuos esto por supuesto es un factor negativo.

La subregión del Ariari, que representa gran parte del Departamento, está conformada por 17 municipios equivalente al 65\% del área departamental y al 5\% del país" (Gomez, Gonzalez, \& Mosquera, 2016, p. 9). Está ubicado el municipio del Dorado Meta, el cual genera un estimado de 2311 toneladas de producción agrícola 
anual, según el Esquema de Ordenamiento Territorial (EOT) residuos de biomasa e inertes (Alcaldía El Dorado, 2012) siendo un indicador y réplica del fenómeno departamental.

El documento de revisión y ajuste del EOT del municipio del Dorado establece que "las zonas rurales del municipio del Dorado no existe ningún tratamiento para los residuos a causa de la inexistencia de una entidad que se encargue de la recolección de éstos" (Alcaldía El Dorado, 2012) determinando la existencia de posibles efectos negativos para el recurso hídrico, atmosférico o el suelo.

El municipio del Dorado es un gran centro agricola por lo que, "se genera gran contenido de residuos agrícolas orgánicos en el lugar, los cuales pueden ser aprovechados, generando productos con valor agregado como abonos orgánicos" (Cacua, 2015, p.91). Adicionando que se pueden utilizar para "hacer una gestión ecológica de los residuos orgánicos, a la vez que se reciclan los nutrientes, de manera que reducen en gran medida la generación de impactos negativos en el ambiente" (Ossa, 2016, p. 34). Dados para "plantear una búsqueda permanente de mecanismos, estrategias y tecnologías capaces de mitigar la pérdida acelerada de los recursos naturales del planeta como alternativa de solución al agotamiento de los recursos naturales" (Avendaño, 2015, p. 20). Por lo anterior se realizo un estudio que permite identificar con herramientas cu

\section{Metodología}

El municipio del Dorado geográficamente está situado en la subregión del Piedemonte llanero, al Noroccidente del departamento del Meta parte alta de la cuenca del río Ariari, que corresponde a la región Orinoquía (Alcaldía del Dorado, 2000), cuenta con un territorio de $115 \mathrm{Km}^{2}$, entre este territorio se pueden apreciar 14 veredas y dos centros poblados. El municipio tiene una población aproximada de 3533 habitantes de los cuales el 63,6\% de la población se encuentra en la zona rural y el 36,4\% en la zona urbana del municipio, del mismo modo cuenta con 783 predios en la zona rural (Alcaldía del Dorado, 2012) figura 1.

\section{Figura 1}

Ubicación geográfica del municipio

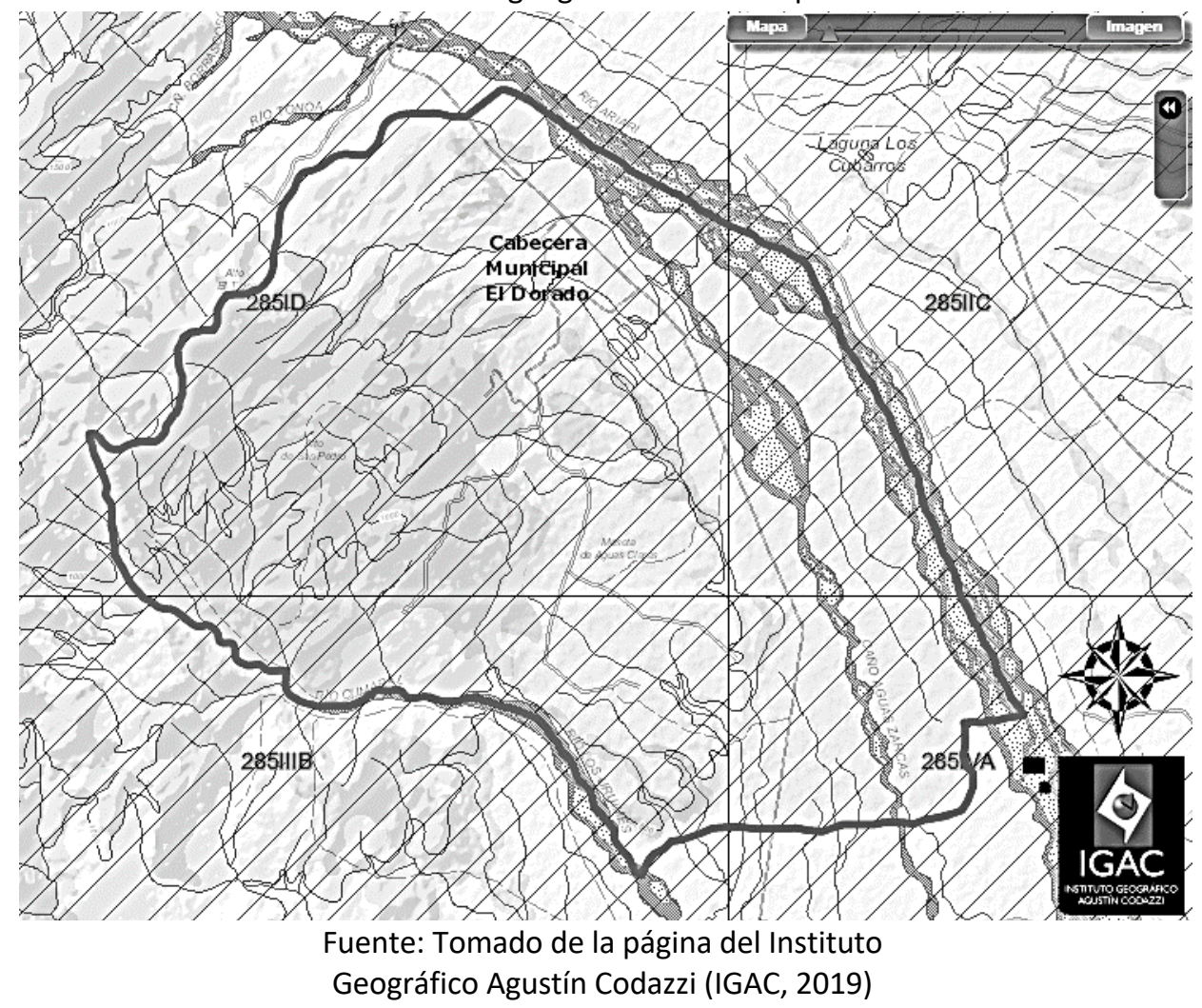


Siendo el municipio representativo en la producción agrícola del departamento del Meta gracias a que "la mayoría de sus habitantes están en el área rural y su principal actividad económica es la agricultura" (Alcaldía del Dorado, 2000) es importante recalcar "la falta de una entidad que se encargue de la gestión de los residuos generados en el área rural del municipio" (Alcaldía del Dorado, 2012).

\subsection{Etapas de la investigación}

Para analizar el impacto ocasionado por las labores agrícolas en el municipio se dividio el trabajo por etapas. En la primera se realizó una identificación de las veredas y sus respectivos cultivos. Así mismo, fue necesario realizar un estudio explicativo, ya que como lo afirma (Hernandez, Fernandez \& Baptista, 2014, p. 23) "se centra en explicar por qué ocurre un fenómeno y en qué condiciones se manifiesta o por qué se relacionan dos o más variables" para determinar a través de la observación los cultivos que mayor residuos generaban y su disposición final.

Como parte de la segunda etapa, se realizó la recolección de datos cuantitativos por medio de una técnica de muestreo probabilístico la cual según (Otzen \& Manterola, 2017, p. 229) "permiten conocer la probabilidad que cada individuo a estudio tiene de ser incluido en la muestra a través de una selección al azar" a través de una encuesta aplicada a todos los agricultores asociados a ASOFRUD (Asociación de fruticultores del Dorado).

Por lo anterior, se validó el instrumento tipo encuesta para la recolección de información con una prueba binomial que consistió a juicio de dos expertos de "una opinión informada de personas con trayectoria en el tema, que son reconocidas por otros como expertos cualificados en éste, y que pueden dar información, evidencia, juicios y valoraciones" (Escobar \& Cuervo, 2008, p. 29). También es importante aclarar que "dicho proceso, consiste en realizar un experimento aleatorio una sola vez y observar si cierto suceso ocurre o no, siendo $p$ la probabilidad de que ocurra (éxito) y $q=1-p$ de que no ocurra (fracaso), por lo que la variable sólo puede tomar dos posibles valores, el 1 si ocurre y el 0 sino sucede. (Martinez \& Marì, 2012, p.5). Es así como la prueba binomial para la variable dependiente se detalla así:

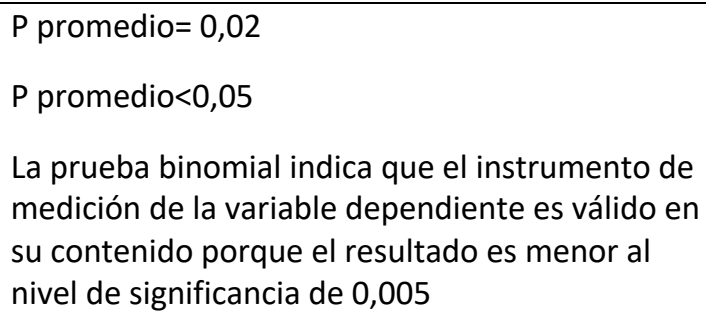

De la misma forma la prueba binomial para la variable independiente se detalla de la siguiente forma:

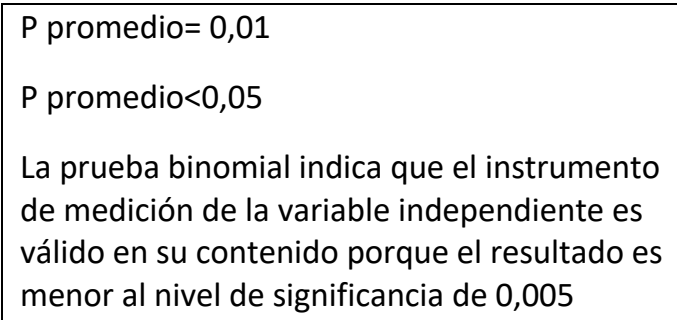

La herramienta permitió recolectar información acerca de los cultivos que más generaban residuos de biomasa "incluye toda la materia viva, o cuyo origen sea la materia viva, que existe en un instante de tiempo en la Tierra" 
(Union Fenosa, 2002) e inertes donde se refieren a "aquellos que no se descomponen ni se transforman en materia prima y su degradación natural requiere grandes períodos de tiempo" (Mora Valencia \& Berbeo Rodríguez, 2010, p. 34) para así ver la relación entre el cultivo, el tipo de residuo que genera y el manejo o proceso que los agricultores le dan a estos.

En una tercera etapa, en donde se procesó la información con el software IBM SPSS statistics 25, que permite "analizar bases de datos para aplicaciones prácticas o para diversas necesidades de investigación" (Castañeda, et al., 2010, p.15). Adicionalmente en una cuarta etapa se analizaron los resultados obtenidos en campo en la matriz de Leopold donde se clasificaron los impactos que se pueden producir, que según (García, 2004, p. 5) "es un método de identificación y puede ser usado como un método de resumen para la comunicación de resultados".

\section{Resultados}

Teniendo en cuenta el instrumento de medición de impactos Matriz de Leopold (Tabla 1), se pudo identificar que hay impacto negativo medio, con un grado de importancia media en lo que respecta al factor físico atmosfera debido a la quema de residuos. (Quintero Núñez \& Moncada Aguilar, 2008, p. 3) especifican que la quema de residuos agrícolas "constituye una fuente importante de emisión de contaminantes al aire, de compuestos como el metano ( $\mathrm{CH} 4)$, monóxido de carbono $(\mathrm{CO})$, bióxido de nitrógeno $\left(\mathrm{NO}_{2}\right)$, hidrocarburos $(\mathrm{NMHC})$ y partículas menores a 10 micras (PM10)". Dicho esto, es posible afirmar que los agricultores en el municipio del Dorado no tienen prácticas de aprovechamiento de residuo; dado que no poseen información en cuanto al manejo que se les puede dar y a la ausencia de un servicio de recolección en la zona rural.

Tabla 1

Matriz de impactos Leopold

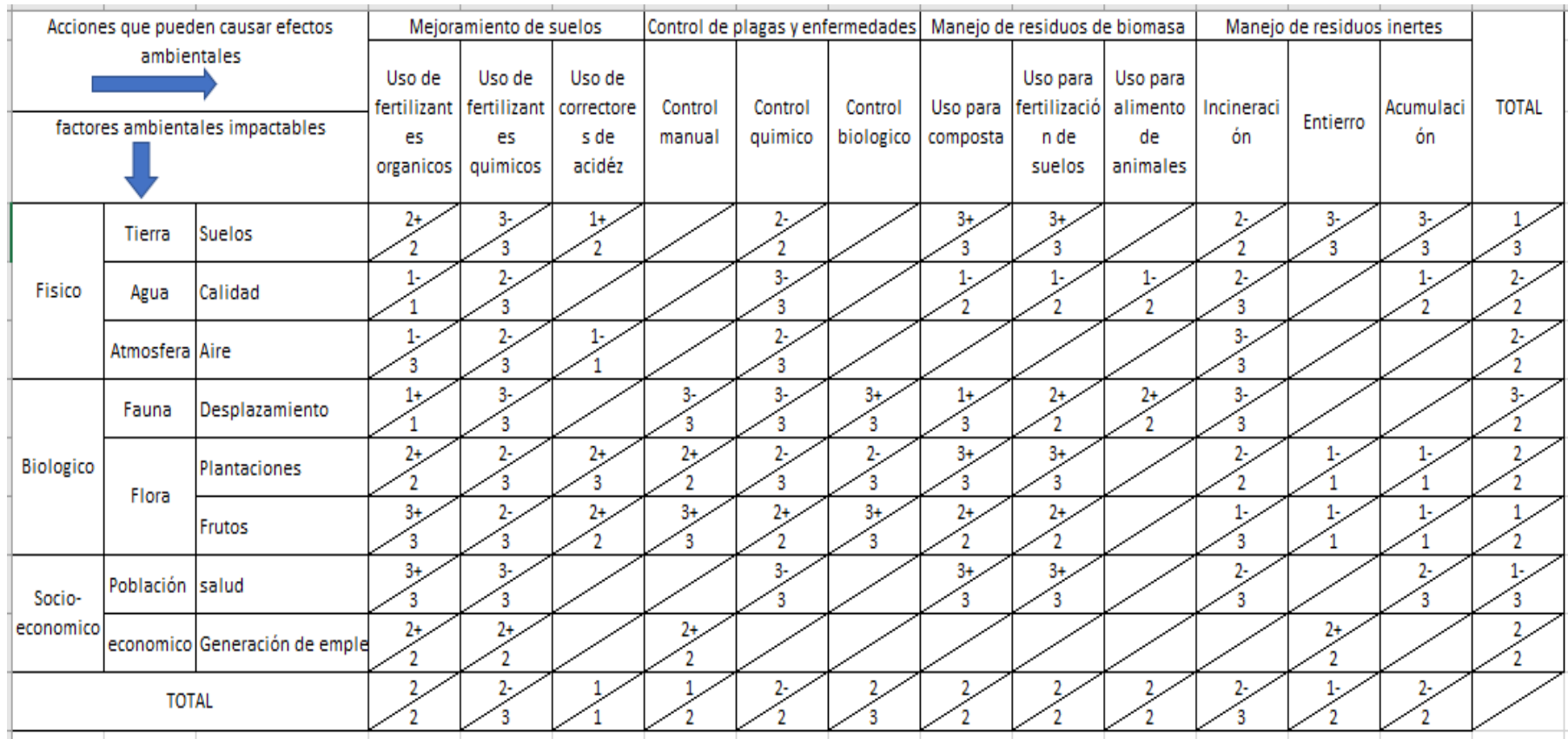

Fuente: Elaboración Propia (2019)

Para lo que se refiere al desplazamiento de fauna, se tiene un impacto negativo alto con una importancia media, pues muchos de los agricultores expresan que, aunque el uso químico para el control de plagas es eficaz, esto conlleva en un detrimento para las especies polinizadoras que ayudan a mantener y reproducir adecuadamente sus cultivos; del mismo modo (Devine, et al, 2008, p.82) en su investigación uso de insecticidas: contexto y consecuencias ecológicas comentan que "los artrópodos que no son el objetivo, se ven a menudo gravemente 
afectados por el uso de insecticidas... Dichos efectos por lo general producen una reducción momentánea de la fertilidad y productividad del suelo". Dando así la razón a los efectos negativos que se pueden dar en los cultivos del municipio. En muchos aspectos, se "reconoce en la agricultura la capacidad de ejercer múltiples funciones para el desarrollo. Dichas funciones tienen que ver con el crecimiento económico" (Perfetti, et al, 2013, p.13); Del mismo modo, en el municipio del Dorado y según la matriz de Leopold, es posible afirmar que se tiene un impacto positivo con importancia media en lo que se refiere a lo económico, pues los agricultores son generadores de empleo.

Con respecto al uso que se les dan a los residuos de biomasa en los cultivos se encontró que el 60,7\% de encuestados los usa para la fertilización de suelos, sin embargo, de este porcentaje, el 37,2\% también utiliza fertilizantes químicos para el mejoramiento de los suelos en sus cultivos; De esta forma y como lo afirma (Alvarez, et al, 2010, p.576) "Un enfoque alterno es usar bajas cantidades de abonos orgánicos y complementar con fertilizantes inorgánicos.". Si bien, esta alternativa es una de las más usadas por los agricultores de la zona, (Cardona, Bolaños, \& Chavarriaga, 2016, p.146) dicen que "Los suelos que recibieron fertilizantes minerales y químicos, presentaron una estructuración deficiente. En contraste...la adición de materia orgánica por medio del compost elaborado con los residuos del sistema agrícola y la incorporación de micorrizas al suelo, permitieron mejorar las propiedades físicas del suelo consideradas en este estudio" lo que podría traducirse en una estructura de suelos en la zona no tan eficiente en comparación de si se utilizara fertilizantes únicamente orgánicos.

De la misma forma, es importante recalcar que en la zona el cultivo que mayor cantidad de residuos genera, con una participación del 79,8\% es el cacao, siendo en un 92,9\% generador de residuos de Biomasa, es así como en el departamento de Caldas se evidencia un comportamiento similar, tal como lo muestra (Corpocaldas, 2015) en su informe "Generación de residuos o desechos peligrosos en el departamento de Caldas" donde es posible apreciar la cantidad anual de residuos producidos por actividad económica, siendo la transformación de cacao responsable de 1659,43 kg. Estableciendo el mismo comportamiento para este cultivo en el municipio del Dorado, dadas las similitudes en cuanto a proporciones, áreas sembradas y manejo, figura 2.

Figura 2

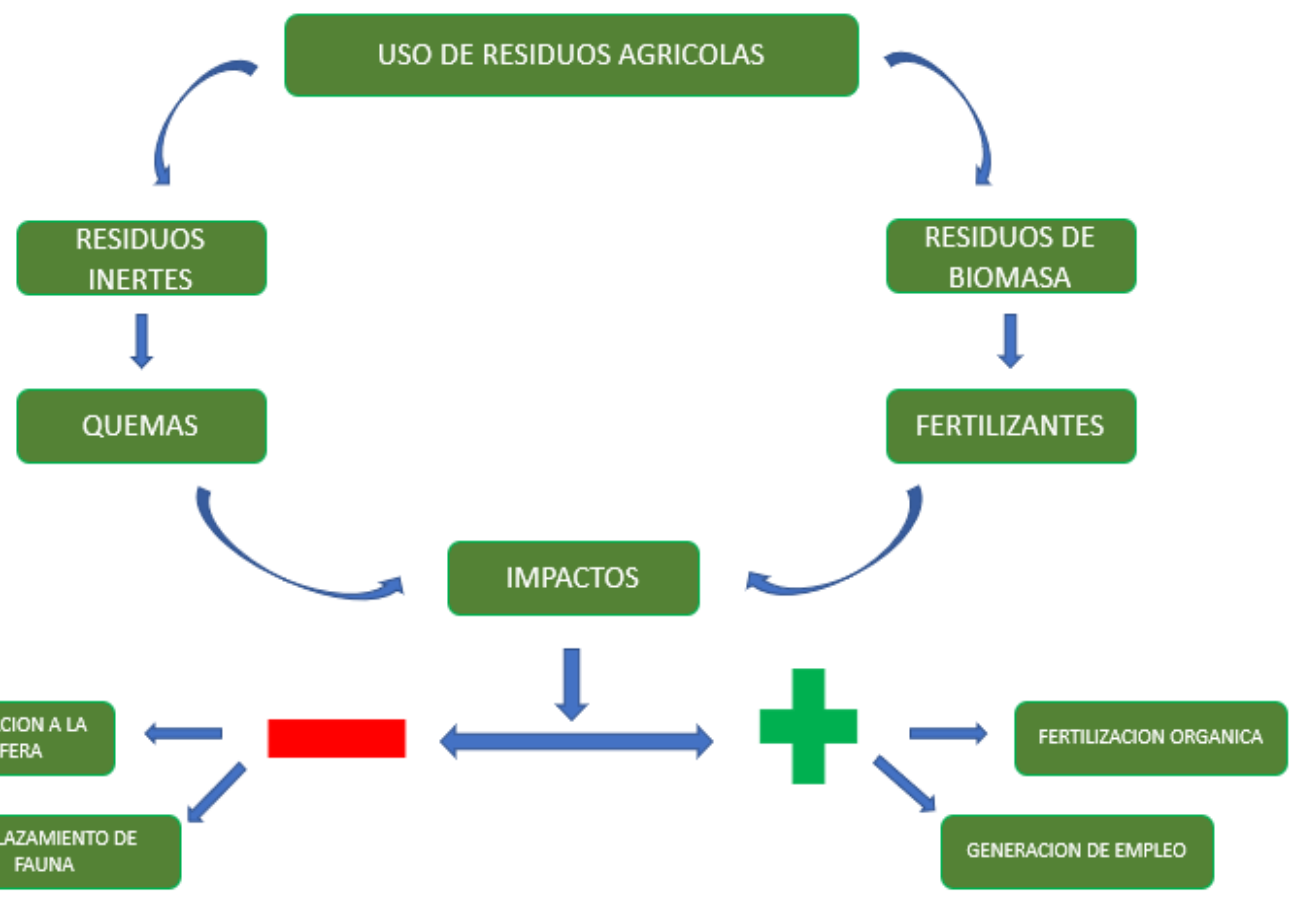

Fuente: elaborado por los autores (2019) 


\section{Conclusiones}

Los residuos inertes son el principal emisor de contaminantes a la atmosfera en dicha zona dado el uso que los agricultores les dan a estos, tales residuos son generadores de gases contaminantes, esto por la ausencia de una entidad recolectora de basuras o por la ausencia de gestión institucional, quienes podrían realizar capacitaciones a los agricultores para mitigar el impacto creando el ambiente para soluciones sustentables.

Del mismo modo, el uso de químicos para el control de plagas disminuye notablemente la fertilidad y productividad del suelo, además del papel que juega en el desplazamiento de polinizadores, quienes no son el objetivo principal afectando la producción de los cultivos, se podría considerar la implementación de cultivos limpios de químicos o el uso de control de plagas biológicos como lo son animales o controles manuales.

Los agricultores no realizan un uso adecuado de los residuos de biomasa como recuperador de suelos, pues aunque la mayoría de estos afirman reusar este tipo de residuos para dicho fin, ya que no le dan un tratamiento adecuado, como es el compostaje para obtener mejores beneficios de la practicas; es de resaltar que un uso inadecuado de estos residuos pueden traer consecuencias para la salud humana, pues generan proliferación de zancudos además de malos olores por el proceso de descomposición de los mismos.

De igual forma el uso de fertilización combinada, puede afectar la estructura de los suelos y generar afectaciones como lo es la disminución de nutrientes y productividad del mismo, es recomendable realizar un solo tipo de fertilización, especialmente el orgánico, ya que se obtienen mejores resultados, convirtiendo el proceso eficiente, disminuyendo los costos de producción.

La zona rural del municipio está constituida por diferentes cultivos, predominando el cacao, el plátano y los cítricos. El primero es el principal generador de residuos de biomasa que pueden ser beneficiados para la elaboración de nuevos subproductos para aprovechar en el cultivo del fruto para dar valor agregado al mismo.

\subsection{Agradecimientos}

El presente trabajo tuvo la colaboración de la asociación de fruticultores del municipio del Dorado (ASOFRUD) quienes prestaron información de total relevancia para dicha investigación, además de contar con la ayuda del señor Luis Antonio Céspedes Murcia, quien tuvo la disposición para guiar e identificar los predios dentro de las veredas del municipio, así como prestar acompañamiento y validar información importante para la misma.

\section{Referencias bibliográficas}

Alcaldía del Dorado A. (2012). Revisión y ajuste del EOT municipio El Dorado Meta 2012 diagnóstico. p.12

Alcaldía del Dorado B. (2000). Esquema de Ordenamiento Territorial municipio El Dorado Meta.

Álvarez, J. D., Gómez, D. A., León N. S., Gutiérrez, F. A. (2010) Manejo integrado de fertilizantes y abonos orgánicos en el cultivo de maíz. Agrociencia, 44 (5), pp. 575-586

Avendaño, E. F. (2015). Panorama actual de la situación mundial, nacional y distrital de los residuos sólidos. Análisis del caso Bogotá D.C. programa basura cero. Trabajo de grado Ingeniero Ambiental: Bogotá D.C Universidad Nacional Abierta y a distancia. pp.20.

Bernal, M. P. \& Gondar, D. (2008). Compostaje, Producción y gestión de los residuos orgánicos: situación actual a nivel mundial, comunitario y estatal: Mundi Prensa Libros S.A. p. 12 
Cacua, L. F. (2015) Análisis del manejo de residuos sólidos agrícolas en la Nueva Sexta, Cúcuta, Norte de Santander: una propuesta de mejoramiento ambiental. Trabajo de grado Magister en desarrollo sostenible y medio ambiente. Manizales; Universidad de Manizales, p. 91.

Cardona, W. A., Bolaños, M. M., Chavarriaga, W. (2016) Efecto de fertilizantes químicos y orgánicos sobre la agregación de un suelo cultivado con Musa acuminata AA. Acta Agron. 65 (2) p. $144-148$

Castañeda, M., Cabrera, A., Navarro Y., Wietse V., (2010) Procesamiento de datos y análisis estadísticos utilizando SPSS. Porto Alegre, p. 15

Coronado, R., \& Valencia, R. (2015). Gestión integral de residuos agrícolas para la generación de materias primas en el municipio de Cota Cundinamarca. Trabajo de grado Ingeniero Agroindustrial: Bogotá D.C Universidad Distrital Francisco José de Caldas, p.14.

Corpocaldas. (2015). Informe de generación residuos o desechos peligrosos en el Departamento de Caldas periodo de balance.

Cuadros, S. (2008). Contaminación de residuos, residuos agrícolas, forestales y lodos, EOI escuela de negocios. p.5

DANE- Departamento Administrativo Nacional de Estadística, (2016). Encuesta Nacional Agropecuaria, Bogotá.

Devine, G., Eza D., Ogusuku E., Furlong M. (2008) Uso de insecticidas: contexto y consecuencias ecológicas. Rev Peru Med Exp Salud Publica. 25(1): 74-100

Escobar, J. \& Cuervo, A. (2008) Validez de contenido y juicio de expertos: una aproximación a su utilización. Avances en Medición, Vol 6, p.27-36.

Figueredo, M., Bello, A., Piedra, A. \& Diez, M. (2011) Evaluación del uso de residuos agrícolas como biofumigantes en el control de nematodos. Centro Agrícola, p.15-19.

García, L. (2004). Aplicación del análisis multicriterio en la evaluación de impactos ambientales. Trabajo de grado doctoral de ingeniería ambiental. Barcelona, Universidad Politécnica de Cataluña, p. 5

Gómez, J., González, K., Mosquera, L. (2016). Competitividad e innovación en el sector frutícola del departamento del Meta. Trabajo de grado Administrador De Empresas. Villavicencio, Universidad de los llanos. p. 9.

Grande, C. (2016). Valoración, biotecnológica de residuos agrícolas y agroindustrial. Editorial Bonaventuriana. p.13. ISBN: 978-958-8785-81-3

Hernandez, R., Fernandez, C. \& Baptista, M. (2014) Metodologia de la investigación 6ta edición, Ed. Mac Graw Hill, Num 136, ISBN: 978-1-4562-2396-0

Lopez, M. \& Boluda, R. (2014). Residuos orgánicos y agricultura intensiva III. 1 (Vol. 1). Ediciones Paraninfo, SA.

Martínez, M., Mari Belloch, M. (2012) La distribución binomial, universidad politécnica de valencia, España. p. 1-8

Mora Valencia, C. A., \& Berbeo Rodríguez, M. L. (2010). Manual de Gestión Integral de Residuos. Bogotá DC: República de Colombia Instituto Nacional de Salud.

Nuñez, D. (2012) Uso de residuos agrícolas para la producción de biocombustibles en el departamento del Meta. Tecnura, 16 (34), p.142-156. 
ONU- Organización de las Naciones Unidas (2011). Paz, dignidad e igualdad en un planeta sano, Población. recuperado el 12 de junio de 2020, https://www.un.org/es/sections/issues-depth/population/index.html.

Ossa, L.(2016). Aplicación de la tecnología de las Pacas Biodigestoras para el tratamiento ecológico de los residuos orgánicos de la Universidad de Antioquia. Tesis de Grado. Facultad de ingeniería. Universidad de Antioquía. Medellín, Colombia.

Otzen, T. \& Manterola, C. (2017) Técnicas de Muestreo sobre una Población a Estudio. Int. J. Morphol, Vol. 35 p. $227-232$

Perfetti, J., Balcázar, Á., Hernández, A., Leibovich J., (2013) Políticas para el desarrollo de la agricultura en Colombia, La Imprenta Editores S.A, Bogotá.

Piña, A., Nieto, D., \& Robles, F. (2016). Utilización de residuos agrícolas y agroindustriales en el cultivo y producción del hongo comestible Seta (Pleurotus spp). Revista Internación de Contaminación Ambiental , 142-151.

Porras, Á. C., \& González, A. R. (2016). Aprovechamiento de residuos orgánicos agrícolas y forestales en Iberoamérica. Academia y virtualidad, 9(2), 90-107.

Quintero Núñez, M., \& Moncada Aguilar, A. (2008). Contaminación y control de las quemas agrícolas en Imperial, California, y Mexicali, Baja California. Región y sociedad, 20(43), 3-24.

Union Fenosa. (2002). Biomasa, un recorrido de la energia. Madrid: E.i.S.E. Domènech, S.A 